\section{Avaliação de diretrizes clínicas brasileiras em oncologia: carências no rigor do desenvolvimento, aplicabilidade e independência editorial}

\author{
Assessment of Brazilian clinical guidelines in \\ oncology: gaps in drafting, applicability, and \\ editorial independence
}

\section{Evaluación de directrices clínicas brasileñas en oncología: carencias en el rigor del desarrollo, aplicabilidad e independencia editorial}

\section{Resumo}

A ampliação da variedade de diretrizes clínicas em oncologia é perceptível em todo o mundo, o que salienta a necessidade de garantir a qualidade destes documentos. Assim, o objetivo do estudo foi avaliar a qualidade de diretrizes nacionais de tratamentos dos cânceres de mama, próstata e de cólon e reto. Foram selecionadas 12 diretrizes brasileiras publicadas por quatro grupos elaboradores distintos (Ministério da Saúde, sistema suplementar de saúde e de sociedades e associações médicas), e aplicado o instrumento AGREE II. Em todas as diretrizes avaliadas foram identificadas fragilidades importantes em mais de um Domínio, com destaque para os baixos valores para "aplicabilidade" e "independência editorial". Os padrões observados por Domínios apresentam-se mais relacionados com o grupo elaborador do que com as condições clinicas tratadas. Menores escores no "rigor do desenvolvimento" e "independência editorial" foram obtidos por grupos elaboradores não governamentais, inclusive com ausência ou falta de transparência nas informações. Mesmo que a "clareza da apresentação" das diretrizes do Ministério da Saúde tenha sido relativamente inferior, na "aplicabilidade" todas apresentaram limitações expressivas. Consequentemente, na avaliação global nenhuma das diretrizes foi recomendada sem modificações, sendo quatro delas não recomendadas. Por fim, é necessário qualificar as recomendações no que tange as evidências que as fundamentam ("rigor metodológico"), assim como dispor de forma compreensivel e exequível as condutas a serem adotadas ("aplicabilidade") $e$ mitigar interesses conflitantes, para que seja ofertado o melhor cuidado aos pacientes oncológicos no país.

Guia de Prática Clínica; Oncologia; Avaliação da Tecnologia Biomédica
Thais Piazza 1

Jans Bastos Izidoro 2

Marcos André Marques Portella Portella 3

Ulysses Panisset 1

Augusto Afonso Guerra-Júnior 4

Mariangela Leal Cherchiglia 1

doi: 10.1590/0102-311X00031920

\author{
Correspondência \\ T. Piazza \\ Pós-graduação em Saúde Pública, Faculdade de Medicina, \\ Universidade Federal de Minas Gerais. \\ Av. Prof. Alfredo Balena 190, sala 729, Belo Horizonte, MG \\ 30130-100, Brasil. \\ piazzathais@gmail.com \\ 1 Faculdade de Medicina, Universidade Federal de Minas \\ Gerais, Belo Horizonte, Brasil. \\ 2 Secretaria de Estado de Saúde de Minas Gerais, Belo \\ Horizonte, Brasil. \\ 3 Hospital Alberto Cavalcanti, Fundação Hospitalar do Estado \\ de Minas Gerais, Belo Horizonte, Brasil. \\ 4 Faculdade de Farmácia, Universidade Federal de Minas \\ Gerais, Belo Horizonte, Brasil.
}




\section{Introdução}

Diretrizes clínicas são documentos compostos por recomendações que visam a otimizar o cuidado ao paciente com determinada condição por profissionais de saúde. Para tal, as diretrizes devem ser baseadas principalmente em revisões sistemáticas com as melhores evidências 1, conhecimentos que não se restringem a apenas resultados de ensaios clínicos ou dados quantitativos 2 . O crescimento da quantidade de diretrizes clínicas publicadas por diferentes instituições ao longo dos anos, somado a contradições entre tais documentos, gerou preocupação quanto à qualidade destas diretrizes 1,3.

Vários países do mundo apresentam diretrizes governamentais que orientam o diagnóstico, tratamento e monitoramento de cânceres 4,5 , além de outras produzidas por organizações não governamentais como sociedades médicas 6,7, o que ocorre de forma semelhante no Brasil. Os documentos produzidos pelo Ministério da Saúde, que são baseados em evidência científica e que visam a nortear as melhores condutas na área da Oncologia no Sistema Único de Saúde (SUS), são chamados de Diretrizes Diagnósticas e Terapêuticas (DDT). Em função da peculiaridade dos procedimentos e tratamentos em oncologia, as DDT não se delimitam apenas às tecnologias incorporadas no SUS, mas sim ao que pode ser proporcionado ao paciente, considerando o possível financiamento repassado (via APAC - Autorização de Procedimentos de Alta Complexidade, conforme a regulamentação pertinente a cada procedimento 8) e a autonomia dos centros que oferecem o cuidado na escolha da melhor opção para cada situação clínica 9 .

Fomentado pela conjuntura mundial de efervescência de diretrizes clínicas, em 2003 o instrumento AGREE (Appraisal of Guidelines for Research es Evaluation) foi criado por uma colaboração internacional entre elaboradores de diretrizes e pesquisadores. O AGREE teve como objetivo avaliar o rigor metodológico e transparência com que diretrizes clínicas são desenvolvidas. A aplicação desse instrumento, em um estudo de Burgers et al. 10, avaliou 100 diretrizes clínicas publicadas entre os anos de 1992 e 1999, originadas de 13 países. Nesse estudo foi verificado que as diretrizes clínicas oncológicas apresentaram maiores escores no "rigor do desenvolvimento" do que as diretrizes para outras condições crônicas. Entretanto, apenas três das 32 diretrizes oncológicas analisadas atingiram, em mais de três dos Domínios avaliados, pontuações superiores a $60 \% 10$.

Posteriormente, em 2009, dado o desenvolvimento contínuo para garantir as propriedades de mensuração, aplicabilidade e viabilidade, o instrumento foi atualizado gerando o AGREE II 11,12. Foi perceptível que a difusão desse instrumento por meio da tradução em diversas línguas se concretizou no crescente número de publicações registradas em bases de dados que a utilizaram em sua metodologia 12. Contudo, ainda são escassos os estudos na literatura científica que avaliaram as diretrizes brasileiras 13,14, sendo que nenhum deles foi voltado especificamente para tratamentos em oncologia. Considerando esse contexto, o objetivo do presente trabalho foi avaliar a qualidade de diretrizes nacionais de tratamentos de cânceres de mama, de próstata e de cólon e reto.

\section{Metodologia}

Foram selecionadas diretrizes brasileiras para os três tipos de câncer mais incidentes (excluindo-se o câncer de pele não melanoma) no Brasil, sendo eles o câncer de mama, de próstata e de cólon e reto ${ }^{15}$. Assim, foi realizada a pesquisa em sítios eletrônicos de instituições nacionais em oncologia, que envolvessem desde o SUS, a iniciativa privada e as associações e sociedades profissionais. Para cada instituição foi verificada a disponibilidade, na forma de acesso livre às diretrizes clínicas oncológicas pesquisadas. Nas situações em que existisse mais de uma diretriz para a mesma condição clínica, foi priorizada a seleção da mais recente com conteúdo relacionado ao tratamento medicamentoso da doença. $\mathrm{O}$ intuito foi garantir a representatividade das diretrizes em língua portuguesa, cujos profissionais de saúde do Brasil têm acesso livre e que potencialmente são as mais recorridas para estudos e consultas. Para cada diretriz selecionada foi procedida a busca por documentos de apoio (informações sobre o processo de desenvolvimento do documento e grupo elaborador) até a data de 30 de agosto de 2018, conforme recomendado pelo instrumento AGREE II 16.

Juntamente com as diretrizes, tais materiais adicionais foram disponibilizados aos avaliadores individualmente por meio de plataforma de elaboração e disponibilização de formulários vir- 
tuais SurveyMonkey (https://pt.surveymonkey.com/), com base na transcrição do instrumento validado 16 e links de armazenamento em nuvem das diretrizes e respectivos documentos de suporte. Como sugerido pelo manual do instrumento 16 e realizado por alguns dos estudos publicados 17,18, avaliadores pertencentes a diferentes grupos de interesse em oncologia realizaram as avaliações em triplicata. Participaram anonimamente cinco pesquisadores(as)/metodologistas, dois médicos(as) oncologistas e cinco gestores(as), sendo mantida a proporção média de profissionais de $56 \%, 22 \%$ e $22 \%$, respectivamente, para cada grupo de diretrizes por tipo de câncer avaliado. Foi orientada a realização do tutorial de treinamento online disponível no sítio eletrônico da iniciativa AGREE 19, assim como a leitura do instrumento na íntegra na versão em português adaptada transculturalmente para o Brasil 16,20.

Todos os avaliadores tiveram acesso às diretrizes e aos respectivos documentos adicionais aos quais ficaram responsáveis, sendo o envio anônimo das respostas (mediante codificação) realizado via plataforma SurveyMonkey. A aquisição de tal interface viabilizou a disposição na íntegra do instrumento AGREE II em sua versão em português (inclusive com as suas observações para cada item, como relacionadas à classificação da completude e o lugar provável para a identificação de cada informação), assim como reforçou a segurança das informações, otimizou a facilidade de preenchimento pelos avaliadores e permitiu estabelecer interface direta para a realização das análises.

O AGREE II é composto por 23 perguntas cuja resposta deve ser fornecida na escala Likert, entre 1 - discordo totalmente até 7 - concordo totalmente, havendo um campo aberto em cada um para observações opcionais. O cálculo das pontuações foi realizado para cada um dos seis Domínios (1 - escopo e finalidade, 2 - envolvimento das partes interessadas, 3 - rigor do desenvolvimento, 4 clareza da apresentação, 5 - aplicabilidade e 6 - independência editorial) em porcentagens, conforme orientado no instrumento 16 , sendo incluídas as respostas dos três avaliadores para cada um dos 23 itens do AGREE II da mesma diretriz. Para a definição da avaliação global (valor da escala entre 1, qualidade mais baixa possível e 7, qualidade mais alta possível e recomendação da diretriz), um dos avaliadores ficou responsável pela harmonização das conclusões fornecidas, definindo pontuação única baseada nas respostas e observações concedidas pelos diferentes avaliadores.

\section{Resultados}

Foram avaliadas 12 diretrizes, quatro por tipo de câncer, cujos grupos elaboradores contemplaram instituições de saúde pública 21,22,23, Associação Médica Brasileira (AMB) em colaboração com a Agência Nacional de Saúde Suplementar (ANS) 24,25,26, instituição privada de saúde 27, assim como de sociedade médica (Sociedade Brasileira de Oncologia Clínica - SBOC) 28,29,30 (Quadro 1). Com relação ao ano de publicação, as diretrizes do câncer de cólon e/ou reto 23,24,25 e as elaboradas pela AMB $24,25,26$ foram relativamente mais antigas.

\section{Domínio 1: escopo e finalidade}

Pode-se notar que, embora sejam diretrizes terapêuticas, existem peculiaridades nas propostas de escopo (Quadro 1). As diretrizes do Ministério da Saúde, Unimed (Confederação Nacional das Cooperativas Médicas) e SBOC apresentaram tratamentos quimioterápicos e radioterápicos. As do Ministério da Saúde preveem adicionalmente o tratamento cirúrgico e também o diagnóstico, e as elaboradas pela AMB são mais restritivas ou quanto ao tipo de tratamento abordado 24,25 ou para um subgrupo da condição clínica 26. Contudo, independentemente da proposta de escopo definida pela diretriz, de forma geral a maioria conseguiu apresentar claramente o seu "escopo e finalidade" (Figura 1 e Tabela 1), com destaque para as elaboradas pela AMB 24,25,26 e pelo Ministério da Saúde 21,22,23. Por sua vez, as menores pontuações para esse Domínio foram verificadas nos documentos produzidos pela operadora de plano privado de saúde 27 e pela SBOC 28,29,30. O primeiro caso esteve relacionado ao fato de o objetivo maior estar disposto de formas distintas nas diretrizes da operadora privada de plano de saúde (gestão dos procedimentos prestados vs. tomada de decisão clínica pelos médicos), e o segundo por não dispor o escopo em local de fácil identificação, sendo o mesmo interpretado por 
Quadro 1

Características das diretrizes clínicas nacionais na área de oncologia, selecionadas por tipo de câncer.

\begin{tabular}{|c|c|c|c|}
\hline Tipo de câncer & Grupo elaborador & Ano de publicação & Título da diretriz \\
\hline \multirow{4}{*}{ Mama } & Ministério da Saúde & 2018 & $\begin{array}{l}\text { Diretrizes Diagnósticas e Terapêuticas do Carcinoma de } \\
\text { Mama }\end{array}$ \\
\hline & AMB, ANS, SBC, SBM e FeBrasGO & 2011 & Câncer de Mama: Terapia Endócrina e Terapia Alvo \\
\hline & Unimed & 2016 & Tratativa Oncológica 2016 - Mama \\
\hline & SBOC & 2017 & Carcinoma de Mama \\
\hline \multirow{4}{*}{ Próstata } & Ministério da Saúde & 2016 & $\begin{array}{l}\text { Diretrizes Diagnósticas e Terapêuticas do } \\
\text { Adenocarcinoma de Próstata }\end{array}$ \\
\hline & $\mathrm{AMB}, \mathrm{SBU}$ & 2017 & $\begin{array}{c}\text { Adenocarcinoma Metastático de Próstata Resistente } \\
\text { à Castração }\end{array}$ \\
\hline & Unimed & 2016 & Tratativa Oncológica 2016 - Próstata \\
\hline & SBOC & 2017 & Câncer de Próstata \\
\hline \multirow{4}{*}{ Cólon e/ou reto } & Ministério da Saúde & 2014 & $\begin{array}{l}\text { Diretrizes Diagnósticas e Terapêuticas do } \\
\text { Câncer de Cólon e Reto }\end{array}$ \\
\hline & AMB, ANS e SBC & 2011 & Câncer de Cólon: Tratamento Quimioterápico \\
\hline & Unimed & 2016 & Tratativa Oncológica 2016 - Cólon e Reto \\
\hline & SBOC & 2017 & Adenocarcinoma Colorretal \\
\hline
\end{tabular}

AMB: Associação Médica Brasileira; ANS: Agência Nacional de Saúde Suplementar; SBC: Sociedade Brasileira de Cancerologia; SBM: Sociedade Brasileira de Mastologia; FeBrasGO: Federação Brasileira das Associações de Ginecologia e Obstetrícia; SBU: Sociedade Brasileira de Urologia; SBOC: Sociedade Brasileira de Oncologia Clínica; Unimed: Confederação Nacional das Cooperativas Médicas.

Figura 1

Gráfico de radar dos resultados obtidos na aplicação do instrumento AGREE II por Domínios para as diretrizes nacionais de câncer de mama, câncer de próstata e câncer de cólon e/ou reto por grupo elaborador.

1a) Câncer de mama

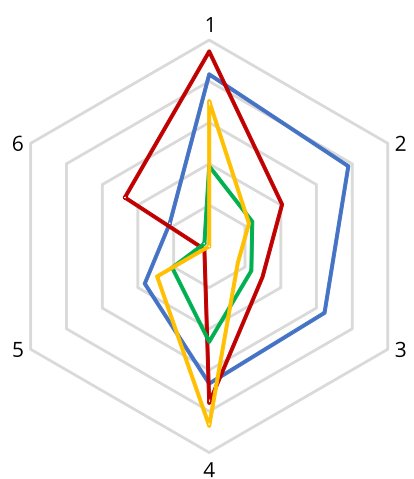

1b) Câncer de próstata

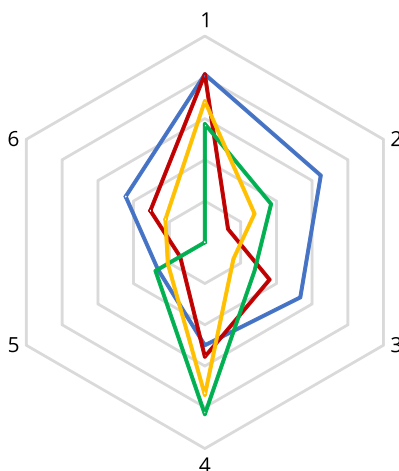

1c) Câncer de cólon e/ou reto

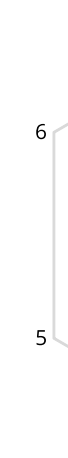

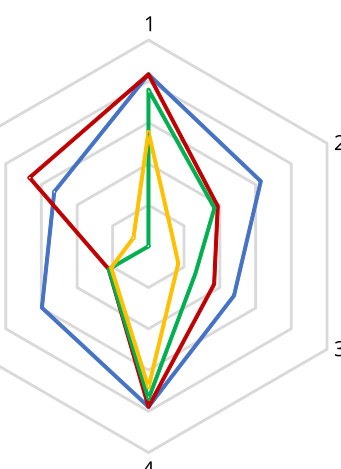

AMB: Associação Médica Brasileira; SBOC: Sociedade Brasileira de Oncologia Clínica; Unimed: Confederação Nacional das Cooperativas Médicas. 
Tabela 1

Resultados dos Domínios e avaliação global da aplicação do instrumento AGREE II para cada diretriz nacional em oncologia selecionada.

\begin{tabular}{|c|c|c|c|c|c|c|c|c|}
\hline \multirow[t]{2}{*}{ Tipo de câncer/Grupo elaborador } & \multicolumn{6}{|c|}{ Domínios (\%) } & \multicolumn{2}{|c|}{ Avaliação global } \\
\hline & 1 & 2 & 3 & 4 & 5 & 6 & $\begin{array}{c}\text { Qualidade global } \\
\qquad(1-7)\end{array}$ & Recomendaria a diretriz \\
\hline \multicolumn{9}{|l|}{ Mama } \\
\hline Ministério da Saúde & 83,3 & 77,8 & 64,6 & 66,7 & 36,1 & 22,2 & 6 & Sim, com modificações \\
\hline AMB, ANS, SBC, SBM e FeBrasGO & 94,4 & 40,7 & 29,9 & 75,9 & 2,8 & 47,2 & 4 & Sim, com modificações \\
\hline Unimed & 38,9 & 24,1 & 23,6 & 46,3 & 20,8 & 2,8 & 3 & Não \\
\hline SBOC & 70,4 & 22,2 & 16,0 & 87,0 & 29,2 & 0,0 & 4 & Sim, com modificações \\
\hline \multicolumn{9}{|l|}{ Próstata } \\
\hline Ministério da Saúde & 81,5 & 64,8 & 53,5 & 50,0 & 26,4 & 44,4 & 5 & Sim, com modificações \\
\hline $\mathrm{AMB}, \mathrm{SBU}$ & 81,5 & 13,0 & 36,1 & 55,6 & 13,9 & 30,6 & 3 & Não \\
\hline Unimed & 57,4 & 37,0 & 27,8 & 83,3 & 27,8 & 0,0 & 3 & Não \\
\hline SBOC & 68,5 & 27,8 & 16,0 & 74,1 & 20,8 & 22,2 & 4 & Sim, com modificações \\
\hline \multicolumn{9}{|l|}{ Cólon e/ou reto } \\
\hline Ministério da Saúde & 83,3 & 63,0 & 47,9 & 77,8 & 59,7 & 52,8 & 4 & Sim, com modificações \\
\hline AMB, ANS e SBC & 83,3 & 38,9 & 36,8 & 77,8 & 22,2 & 66,7 & 3 & Sim, com modificações \\
\hline Unimed & 75,9 & 37,0 & 26,4 & 74,1 & 22,2 & 0,0 & 3 & Não \\
\hline SBOC & 55,6 & 13,0 & 16,7 & 68,5 & 20,8 & 8,3 & 4 & Sim, com modificações \\
\hline
\end{tabular}

AMB: Associação Médica Brasileira; ANS: Agência Nacional de Saúde Suplementar; SBC: Sociedade Brasileira de Cancerologia; SBM: Sociedade Brasileira de Mastologia; FeBrasGO: Federação Brasileira das Associações de Ginecologia e Obstetrícia; SBU: Sociedade Brasileira de Urologia; SBOC: Sociedade Brasileira de Oncologia Clínica; Unimed: Confederação Nacional das Cooperativas Médicas.

exclusão como voltado para o tratamento (menciona que não são objetivos avaliar estadiamento, rastreamento e nem condições fisiopatológicas).

\section{Domínio 2: envolvimento das partes interessadas}

Os itens menos pontuados no Domínio 2 (Figura 1 e Tabela 1) estão relacionados a não consideração das opiniões da população-alvo (pacientes/usuários), assim como o fato das diretrizes terem sido elaboradas por um único grupo profissional. A pontuação superior das três diretrizes do Ministério da Saúde 21,22,23 em relação às demais pôde ser evidenciada de forma clara justamente por contar com um grupo elaborador diversificado em relação às demais (produzidas majoritariamente por equipes de médicos especialistas).

\section{Domínio 3: rigor do desenvolvimento}

As escalas de valores do Domínio 3 das diretrizes do mesmo grupo elaborador se mantiveram próximas (variação de no máximo de 16,7\%), inclusive permanecendo a posição entre os grupos elaboradores. A superioridade das três diretrizes do Ministério da Saúde, seguida das da AMB, Unimed e SBOC, respectivamente (Figura 1 e Tabela 1), deve-se aos seguintes fatores: acesso aos detalhes da metodologia utilizada nos documentos adicionais das diretrizes da Ministério da Saúde 31,32, informações estas não disponíveis para as outras diretrizes; quando minimamente descrita, a qualidade metodológica muitas das vezes foi questionável 24,25,26,28,29,30; e ausência global de informações sobre os procedimentos de atualização das diretrizes. 
Para este Domínio, verifica-se a variabilidade por tipo de câncer, com destaque para as diretrizes da SBOC 28, seguidas da AMB 24 no câncer de mama da Unimed 27 e SBOC 29 no câncer de próstata, e sem grande variação nas relativas ao câncer de cólon e/ou reto (menor que 10\%) 23,25,27,30. O melhor desempenho ocorreu pela facilidade de identificar as recomendações-chave (uso de trechos resumidos com destaque 24 ou quadro de síntese 28), pela delimitação clara da prática a ser adotada estar especificada por estadiamento do câncer 28,29 , uso de fluxogramas de sequência de tratamentos para duas diretrizes de câncer de próstata 27,29, e por fim por abordar maior variabilidade dos tipos de tratamentos 21,22,23,28.

\section{Domínio 5: aplicabilidade}

Trata-se de um dos Domínios que de maneira geral obteve menor pontuação em todas as diretrizes (Figura 1 e Tabela 1), como as diretrizes para o câncer de próstata da Unimed 27 e Ministério da Saúde 22 que atingiram valores próximos (27,8\% e 26,4\%, respectivamente). Entretanto, as diretrizes para os cânceres de mama e cólon e reto do Ministério da Saúde 21,23 obtiveram os melhores desempenhos por apresentarem aspectos como a disponibilidade de tratamentos a cargo das unidades que prestam tais tratamentos (como por exemplo, a necessidade de dispor de radioterapia para o tratamento do câncer retal), como também por considerarem as consequências na sobrevida e custo-efetividade das tecnologias. No caso da diretriz para câncer de próstata da Unimed 27, a questão dos recursos financeiros foi abordada em diversos momentos (inclusive citando a possibilidade de negociação de pacotes com a justificativa em casos de recusas de cobertura), mas as fontes de informações, os dados e as metodologias aplicadas para subsidiar os posicionamentos não foram apresentados. Ainda sobre as mesmas diretrizes da Unimed 27 , as próprias câmaras técnicas envolvidas com o desenvolvimento da tratativa são responsáveis pela auditoria e implementação dos procedimentos (exemplos de ficha de requisição no documento).

\section{Domínio 6: independência editorial}

O Domínio 6, assim como o 5, obteve globalmente a menor pontuação em todas as diretrizes, existindo alternância no posicionamento dos grupos elaboradores por tipo de diretriz em relação a este quesito (Figura 1 e Tabela 1). Tal variação pode ser explicada pelas diretrizes da AMB 24,25,26 conterem em suas partes introdutórias a informação "nenhum conflito de interesse declarado" (embora sem justificativa detalhada) e por sua vez as do Ministério da Saúde 21,22,23 terem pontuado comparativamente melhor na questão relativa à influência do órgão financiador sobre os conteúdos das diretrizes. Para as três condições de saúde, as diretrizes da Unimed 27 apresentaram o pior desempenho, pois além de não informarem conflitos de interesses, a equipe elaboradora também desempenhava simultaneamente cargos de gestão na cooperativa, somado ao fato de embora não informar a influência do órgão financiador sobre os conteúdos, em diversas partes das diretrizes a necessidade de otimizar gastos foi mencionada.

\section{Avaliação global}

A maioria das diretrizes avaliadas $21,23,24,25,28,29,30$ foi considerada recomendada segundo a avaliação realizada, mas necessita de modificações, e quatro não foram recomendadas 26,27 . As variações nos Domínios observadas entre as diretrizes foram, de forma geral, maiores entre os grupos elaboradores do que entre as diferentes diretrizes do mesmo grupo elaborador. Esse panorama condiz com o esperado, visto que as informações referentes ao processo de desenvolvimento das diretrizes e a estrutura de apresentação do documento por cada grupo são as mesmas ou muito similares. 


\section{Discussão}

A avaliação de diretrizes em oncologia brasileira, produzidas por grupos elaboradores diversos (diretrizes do SUS, do sistema suplementar e de sociedades e associações médicas), revelou que existem fragilidades consideráveis em mais de um Domínio em todos os documentos. Pondera-se que a representatividade foi buscada tanto no que tange as entidades que formularam as diretrizes, assim como na seleção entre os tipos de cânceres mais incidentes no país, os quais, em tese, teriam maior atenção e engajamento na elaboração de diretrizes significativas tanto no setor público quando no privado.

Como verificado em um estudo que avaliou as diretrizes clínicas brasileiras de diferentes grupos elaboradores para doenças não transmissíveis relevantes no âmbito da atenção primária 13 , nenhuma das diretrizes nacionais em oncologia declarou as fontes de financiamento ou citou ter realizado a adaptação de outra diretriz para a sua elaboração. Ainda, de forma semelhante ao verificado em outros estudos, nenhuma delas foi recomendada sem modificações 13,14. Embora o instrumento AGREE II também tenha o potencial de ser usado como checklist durante a elaboração de diretrizes para favorecer a qualidade, e não só para avaliá-la após publicadas 33 , nenhuma das diretrizes estudadas informou ter recorrido ao instrumento nessa etapa. Por sua vez, existem exemplos de grupos elaboradores de diretrizes que utilizam esse instrumento, como o sistema de saúde colombiano nos Guías de Práctica Clínica 34 e o Scottish Intercollegiate Guidelines Network (SIGN), mesmo que este último recorra à versão inicial do AGREE 35.

O Domínio "escopo e finalidade" foi o mais bem pontuado em todas as diretrizes, como verificado em outros estudos que avaliaram diretrizes brasileiras $13,14 \mathrm{e}$ internacionais 36,37 . E embora o principal objetivo das diretrizes clínicas seja melhorar a qualidade do cuidado provido 1,2, percebe-se que as diretrizes brasileiras em oncologia se propõem a atender outros tipos de demandas, como reunir todas as práticas disponíveis para determinada condição, e definir normas para uso de procedimentos no contexto ao qual está inserido com um foco mais explícito em racionalização de recursos.

Além das diretrizes do Ministério da Saúde, nenhuma fez referência a tratar-se de atualização da versão anterior ou mencionou de alguma forma possíveis atualizações futuras. Aparentemente, para além do critério legal de periodicidade na atualização a cada dois anos para as diretrizes do Ministério da Saúde 38, estão outras prioridades. Recentemente a divulgação da lista Protocolos e Diretrizes em atualização, elaboração ou análise no site da Conitec (Comissão Nacional de Incorporação de Tecnologias no SUS) 39 foi um avanço, embora os critérios para a priorização não estejam dispostos explicitamente. No caso da diretriz do Ministério da Saúde para o câncer de cólon e reto não estava prevista uma nova publicação, como constatado na lista de diretrizes que estão no processo de atualização, atualizada em 30 de abril de 2020. Por outro lado, as outras diretrizes do Ministério da Saúde 21,22 encontram-se em situações distintas. A do câncer de mama foi a publicada mais recentemente 21 e já está sendo submetida à segunda atualização 39 , e a do Adenocarcinoma Prostático também não consta na lista de previsão de atualização. Tal fato chama a atenção pois, para essa condição clínica, ocorreu a desincorporação do medicamento ciproterona em pacientes resistentes à castração $40 \mathrm{e}$ a incorporação da abiraterona para este perfil de pacientes que já tivessem realizado quimioterapia 41.

Com relação ao "envolvimento das partes interessadas" (Domínio 2) e "rigor do desenvolvimento" (Domínio 3), a observação dos melhores desempenhos para as diretrizes produzidas por agências governamentais em relação às de sociedades e associações médicas concorda com a literatura 12,42. Embora as diretrizes do Ministério da Saúde tenham tido destaque comparativamente, tais Domínios ainda podem ser melhorados 14 por estarem em torno de $65 \%$ e 50\%, respectivamente. Mesmo com a publicação do detalhamento do processo de elaboração das diretrizes 31 , a Conitec, até junho de 2016, realizou aproximadamente $1 / 3$ dos seus processos de forma simplificada (sem consulta pública), além de ser composta majoritariamente por membros do Ministério da Saúde e dependente hierárquica e orçamentariamente deste Ministério (diferente de agências de avaliação de tecnologia em saúde - ATS - em outros países) 43 . Com relação aos documentos internacionais analisados, 67 diretrizes publicadas entre 2005 e 2013 apresentaram resultados semelhantes ao do presente estudo (Domínio 3 com 64\%) 44, assim como nas 124 diretrizes da Organização Mundial da Saúde (OMS) (55\% no Domínio 2 e 56\% no 3) 39, e 48 diretrizes estabelecidas por uma aliança de centros oncológicos dos Estados Unidos (National Comprehensive Cancer Network - NCCN) internacionalmente reconhecida (48,6\% no Domínio 2 e 62,4\% no 3) 45. 
Especificamente no caso do Domínio 3 ("rigor do desenvolvimento"), o uso de informações provenientes de outros tipos de publicações que não ensaios clínicos é limitado nas diretrizes avaliadas. Isso pode estar intimamente relacionado à dificuldade no julgamento e capacidade de integração de dados, em especial para os qualitativos. Embora existam métodos capazes de congregar tais informações, ainda são ferramentas com características majoritariamente frequentistas 2. Um exemplo de ferramenta internacionalmente reconhecida para a avaliação de recomendações é o sistema GRADE (Grading of Recommendations, Assessment, Development and Evaluation) 46. Esse é capaz de determinar o nível da evidência e o grau de recomendação, considerando a diversidade de informações resgatadas e por isto a sua aplicação vem sendo incorporada durante a elaboração de diretrizes mundialmente, como no caso das diretrizes da OMS (aproximadamente metade vem empregando o GRADE) ${ }^{37} \mathrm{e}$ nos sistemas de saúde do Reino Unido 35,47 e Colômbia ${ }^{34}$. Contudo, para as diretrizes brasileiras, apenas as do Ministério da Saúde aplicaram a referida ferramenta explicitamente, a partir de 201631. Observou-se o uso de definições próprias de classificação das recomendações, somado ao fato de não estarem caracterizadas claramente, o que gera dificuldade de compreensão, reprodutibilidade e do estabelecimento de comparações, seja entre as diretrizes avaliadas $24,25,26,28,29,30$ ou com outras, como as internacionais. Embora apresentem maior detalhamento nas classificações das recomendações e níveis de evidência adotados, é frequente que diretrizes internacionais também estabeleçam especificações próprias 6,7, ainda que testem o GRADE mantendo a sua classificação própria de recomendação (caso da diretriz vigente do câncer de mama publicada em 2013 pela SIGN 35).

Não foi verificada, nas diretrizes brasileiras em oncologia, o uso nem a descrição de processo para o estabelecimento de consenso entre os especialistas durante a etapa de desenvolvimento, ainda que previsto na metodologia 31 . Esse fato chama a atenção, pois embora não seja sempre detalhadamente descrita, é parte metodológica vastamente adotada 42,48 . Seu uso permite definir prioridades em situações específicas, como também combinar o conhecimento de forma contextualizada com a prática profissional 2. Entretanto, é pertinente considerar o estudo de documentos internacionais feito por Jacobs et al. 44, que avaliou, além de diretrizes, 34 declarações de consenso de grupos de especialistas. Foi constatada a inferioridade no "rigor do desenvolvimento" desses documentos (diferença média de $32 \%$ ) em relação às diretrizes clínicas, além da menor "independência editorial" (inferior em 15\% na diferença média) 44 . Constata-se assim que o relato do processo decisório do estabelecimento das recomendações está muitas vezes ausente não só em diretrizes nacionais. Tal situação confere gravidade, pois permite supor ou a não realização do processo de consenso de fato, ou ainda, a opção da não exposição dessa etapa na estruturação de diretrizes.

Sobre o Domínio 4, que trata da "clareza da apresentação", as diretrizes do Ministério da Saúde apresentaram pontuações inferiores comparado a outras diretrizes nacionais 13 e da OMS (média de 91\%) 37. Isso pode estar intimamente relacionado com as características peculiares de flexibilidade da terapêutica oncológica em relação a outras doenças 8,9, que geralmente não estabelecem condutas únicas a serem adotadas, ampliando as possibilidades de tomadas de decisão na clínica.

O instrumento AGREE II foi estruturado para conseguir avaliar diretrizes que apresentem qualquer etapa do cuidado em saúde, independentemente da condição clínica, entretanto não faz parte de seu propósito investigar as questões organizacionais do cuidado em saúde 16 . Contudo, é válido ressaltar que existem diferenças entre o escopo e, consequentemente, na aplicação prática das diretrizes em estudo, que vão desde atender a um sistema de saúde universal, plano de saúde privado, ou ainda consensos entre grupos de especialistas. A despeito de realizar a descrição dos facilitadores e barreiras, discutir sobre recursos e questões de implementação e monitoramento do impacto das recomendações serem relevantes, até mesmo no caso das diretrizes da OMS, o Domínio "aplicabilidade" foi o segundo a atingir a menor média (52\%). Isso pode estar relacionado justamente com a dificuldade de atingir a amplitude do propósito a ser contemplado (âmbito da saúde pública global) 37, embora já tenha sido verificado que diretrizes elaboradas por sistemas de saúde sem fins lucrativos estejam mais associadas ao melhor desempenho na "aplicabilidade", quando comparadas a diretrizes de outras origens 49 . Nesse sentido, cabe uma reflexão sobre as ferramentas que podem estimular a aplicação prática das recomendações de diretrizes, como o uso de instrumentos educativos, documentos resumidos, guias e/ou ferramentas digitais. Essas não foram exploradas nas diretrizes avaliadas, embora internacionalmente existam iniciativas crescentes neste sentido, como: a apresentação de diferentes versões das diretrizes colombianas (completa, para profissionais de saúde e para cuidadores) 34; o 
MagicApp (MAGIC Evidence Ecosystem Foundation. https://app.magicapp.org/), aplicativo elaborado e continuamente atualizado por equipe internacional de médicos e pesquisadores com o objetivo de otimizar a adaptação, a apresentação e a disseminação de diretrizes; e a Soft Systems Methodology (SSM), ainda que pouco utilizada 50. Esse último é um processo estruturado, embora flexível, que considera a natureza multidimensional complexa do contexto e cultura locais, inclusive a possibilidade de opiniões divergentes entre as partes interessadas, para então manejar adequadamente o cuidado em saúde 50.

Mesmo em países que dispõem de diretrizes clínicas em oncologia internacionalmente reconhecidas e consideradas de alta qualidade, verifica-se a prática de tratamentos discordantes das recomendações preconizadas, inclusive com a realização de intervenções terapêuticas desnecessárias. Isso gera consequências clínicas e econômicas desfavoráveis para o sistema de saúde 51,52. No Brasil, foi constatado o cenário desigual no acesso aos tratamentos oncológicos no SUS 53, a demanda pela incorporação de novas tecnologias e também a pressão pelo registro de novas alternativas terapêuticas em um menor período de tempo 54. Internacionalmente consequências vêm sendo observadas, como no caso dos medicamentos oncológicos com registro acelerado pelo Administração de Alimentos e Medicamentos dos Estados Unidos (U.S. Food and Drug Administration - FDA) nos últimos anos 55,56. A efetividade observada tem se apresentado muito inferior à eficácia verificada em ensaios clínicos 56, além de ter havido aumento do número de produtos que precisaram ser retirados do mercado 55. Pelos motivos apresentados, aplicar de forma concreta diretrizes de qualidade 1 é imprescindível não só para a melhoria no desempenho do Domínio "aplicabilidade" 13, mas também para garantir a sustentabilidade de sistemas de saúde e prestação do melhor cuidado em saúde às pessoas. Algumas estratégias que podem ser recorridas nesse sentido envolvem tanto melhorar a disseminação das informações das diretrizes (como publicações de resumos, uso de fluxogramas e destaque para as recomendações-chave) quanto monitorar a aplicação das mesmas (instruções para a implementação e uso de indicadores, por exemplo) 16 .

Mesmo com valores maiores no Domínio "independência editorial" em relação ao perfil das diretrizes brasileiras em oncologia (com médias de $68 \% 44,56 \% 48,48 \% 37$, por exemplo), a literatura concorda com a falta das declarações de conflitos de interesses ou ainda, quando presentes, frequentemente não estão explícitas. Essa situação coloca a integridade e a qualidade das diretrizes em dúvida sob o prisma da isenção.

É necessário considerar a subjetividade inerente ao instrumento AGREE II. As diferenças entre os valores atribuídos pelos avaliadores estão relacionadas ao nível de julgamento individual, o que é intrínseco a tal processo para o qual não existe resposta esperada, mas que simultaneamente congregam diferentes perspectivas sobre as diretrizes avaliadas 16 . Somado a isso, ao longo da realização deste trabalho ocorreu a atualização da diretriz do Ministério da Saúde para o câncer de mama ${ }^{57}$. Entretanto, com base na observação de que a única alteração significativa foi eximir a necessidade de teste molecular para confirmar a indicação do trastuzumabe às mulheres com três cruzes na imuno-histoquímica para HER-2. Portanto, não ocorreram alterações nos esquemas terapêuticos propriamente ditos; observamos inclusive que a redação da metodologia permanece a mesma da versão anterior 21 .

Também é necessário mencionar que é notória a variabilidade de parâmetros na definição de um ponto de corte aceitável, ou sua ausência 45 , para a classificação da qualidade global encontrada na literatura, variando, por exemplo, $70 \%$ ou 4 17. Por isso, optou-se por não adotar valor preestabelecido. Nesse quesito abrangente, nenhuma das diretrizes brasileiras em oncologia foi recomendada sem modificações, sendo quatro delas não recomendadas 26,27 , as mesmas caracterizadas pelos baixos escores nos Domínios de "rigor do desenvolvimento", "aplicabilidade" e "independência editorial". 


\section{Conclusão}

O descolamento entre o conteúdo das diretrizes nacionais em oncologia e o que cada um dos seis Domínios constantes no AGREE II contemplam foi verificado claramente. Ao passo que as diretrizes do Ministério da Saúde apresentaram maior rigor metodológico e participação de diferentes atores no processo de elaboração, as referidas diretrizes mostraram fraco desempenho na clareza e execução prática das recomendações. Por sua vez, as diretrizes originadas de grupos médicos especializados, embora tenham tido apresentação mais clara no fornecimento da recomendação, deixaram à parte aspectos metodológicos relevantes, assim como as questões relacionadas aos conflitos de interesses de seus elaboradores. Mesmo considerando a vocação de cada diretriz no âmbito de atuação ao qual se propõe estar inserida (público ou privado), todas apresentaram deficiências. Diretrizes clínicas com recomendações estabelecidas sem suporte de evidências qualificadas, denotando um certo caráter impositivo, somadas à carência de aplicabilidade e associadas às fragilidades na independência editorial, geram grande diversidade de condutas possíveis. Fato que influencia na efetividade das práticas clínicas e, consequentemente, no cuidado aos pacientes oncológicos. Nesse sentido, é necessário investir na transparência e qualificações contínuas no processo de elaboração de diretrizes clínicas em oncologia no Brasil.

\section{Colaboradores}

T. Piazza contribuiu na concepção e projeto, coleta, análise e interpretação dos dados e redação do artigo. J. B. Izidoro e M. A. M. P. Portella contribuíram na coleta e interpretação dos dados, e revisão crítica relevante do conteúdo intelectual. U. Panisset e A. A Guerra-Júnior contribuíram na revisão crítica relevante do conteúdo intelectual e aprovação final da versão a ser publicada. M. L. Cherchiglia contribuiu na concepção e projeto, revisão crítica relevante do conteúdo intelectual, aprovação final da versão a ser publicada.

\section{Informações adicionais}

ORCID: Thais Piazza (0000-0001-9385-7609); Jans Bastos Izidoro (0000-0002-8625-2676); Marcos André Marques Portella Portella (0000-0002-20764679); Ulysses Panisset (0000-0001-9357-8116); Augusto Afonso Guerra-Júnior (0000-0001-52560577); Mariangela Leal Cherchiglia (000-00015622-567X).

\section{Agradecimentos}

À Coordenação de Aperfeiçoamento de Pessoal de Nível Superior (CAPES) por conceder bolsa de estudos para a realização do doutorado da T. Piazza; ao Conselho Nacional de Desenvolvimento Científico e Tecnológico (CNPq) e à Fundação de Amparo à Pesquisa do Estado de Minas Gerais (FAPEMIG) pelo suporte financeiro (processo APQ-03475-13); e a todos os avaliadores que voluntariamente se disponibilizaram para realizar a avaliação das diretrizes em estudo. 


\section{Referências}

1. Institute of Medicine. Clinical practice guidelines we can trust. https://www.ncbi.nlm. nih.gov/books/NBK209539/pdf/Bookshelf_ NBK209539.pdf (acessado em 16/Fev/2020).

2. Wieringa S, Dreesens D, Forland F, Hulshof C, Lukersmith S, Macbeth F, et al. Different knowledge, different styles of reasoning: a challenge for guideline development. BMJ Evid Based Med 2018; 23:87-91.

3. Qaseem A, Forland F, Macbeth F, Ollenschläger G, Phillips S, van der Wees P. Guidelines international network: toward international standards for clinical practice guidelines. Ann Intern Med 2012; 156:525-31.

4. National Comprehensive Cancer Network. NCCN guidelines, 2017. https://www.nccn. org/professionals/physician_gls/ (acessado em 16/Fev/2020).

5. National Institute for Health and Care Excellence. NICE guidance. https://www.nice.org. uk/guidance (acessado em 16/Fev/2020).

6. European Society for Medical Oncology. ESMO clinical practice guidelines. http:// www.esmo.org/Guidelines (acessado em 16/ $\mathrm{Fev} / 2020)$

7. Burstein HJ, Lacchetti C, Anderson H, Buchholz TA, Davidson NE, Gelmon KA, et al. Adjuvant endocrine therapy for women with hormone receptor-positive breast cancer: ASCO clinical practice guideline focused update. J Clin Oncol 2019; 37:423-38.

8. Departamento de Regulação Avaliação e Controle, Secretaria de Atenção à Saúde, Ministério da Saúde. Manual de bases técnicas da oncologia - SIA/SUS - Sistema de Informações Ambulatoriais. https://www.inca.gov.br/sites/ ufu.sti.inca.local/files//media/document//ma nual-oncologia-25a-edicao.pdf (acessado em 16/Fev/2020).

9. Comissão Nacional de Incorporação de Tecnologias no Sistema Único de Saúde. Protocolos e diretrizes. http://conitec.gov.br/protoco los-e-diretrizes (acessado em 09/Mai/2020).

10. Burgers JS, Fervers B, Haugh M, Brouwers M, Browman G, Philip T, Cluzeau FA. International assessment of the quality of clinical practice guidelines in oncology using the appraisal of guidelines and research and evaluation instrument. J Clin Oncol 2004; 22:2000-7.

11. AGREE II - Appraisal of Guidelines Research \& Evaluation. https://www.agreetrust.org/ agree-ii/ (acessado em 16/Fev/2020).

12. Makarski J, Brouwers MC. The AGREE Enterprise: a decade of advancing clinical practice guidelines. Implement Sci 2014; 9:103.

13. Molino CGRC, Romano-Lieber NS, Ribeiro E, Melo DO. Non-communicable disease clinical practice guidelines in Brazil: a systematic assessment of methodological quality and transparency. PLoS One 2016; 11:e0166367.
14. Ronsoni RDM, Pereira CCA, Stein AT, Osanai MH, Machado CJ. Avaliação de oito Protocolos Clínicos e Diretrizes Terapêuticas (PCDT) do Ministério da Saúde por meio do instrumento AGREE II: um estudo piloto. Cad Saúde Pública 2015; 31:1157-62.

15. Instituto Nacional de Câncer José Alencar Gomes da Silva. Estimativa 2020: incidência de câncer no Brasil. https://www.inca.gov.br/si tes/ufu.sti.inca.local/files//media/document// estimativa-2020-incidencia-de-cancer-nobrasil.pdf (acessado em 16/Fev/2020).

16. Consórcio AGREE. Instrumento para avaliação de diretrizes clínicas AGREE II. https:// www.agreetrust.org/wp-content/uploads/ 2013/06/AGREE_II_Brazilian_Portuguese. pdf (acessado em 16/Fev/2020).

17. Barcelo A, Jawed M, Qiang A; PAHO Diabetes Guideline Project Group. A review of the quality of current diabetes clinical practice guidelines. Rev Panam Salud Pública 2017; 41:e90.

18. Font-Gonzalez A, Mulder RL, Loeffen EAH, Byrne J, van Dulmen-den Broeder E, van den Heuvel-Eibrink MM, et al. Fertility preservation in children, adolescents, and young adults with cancer: quality of clinical practice guidelines and variations in recommendations. Cancer 2016; 122:2216-23.

19. AGREE II training tools. http://www.agree trust.org/resource-centre/agree-ii-trainingtools/ (acessado em 16/Fev/2020).

20. Khan GSC, Stein AT. Adaptação transcultural do instrumento Appraisal of Guidelines for Research \& Evaluation II (AGREE II) para avaliação de diretrizes clínicas. Cad Saúde Pública 2014; 30:1111-4.

21. Ministério da Saúde. Portaria Conjunta no 19, de 3 de julho de 2018. Aprova as Diretrizes Diagnósticas e Terapêuticas do Carcinoma de Mama. Diário Oficial da União 2018; 16 jul.

22. Ministério da Saúde. Portaria no 498, de 11 de maio de 2016. Aprova as Diretrizes Diagnósticas e Terapêuticas do Adenocarcinoma Próstata. Diário Oficial da União 2016; 12 mai.

23. Ministério da Saúde. Portaria no 958, de 26 de setembro de 2014. Aprova as Diretrizes Diagnósticas e Terapêuticas do Câncer de Cólon e Reto. Diário Oficial da União 2014; 29 set.

24. Associação Médica Brasileira; Agência Nacional de Saúde Suplementar; Sociedade Brasileira de Cancerologia; Sociedade Brasileira de Mastologia; Federação Brasileira da Associações de Ginecologia e Obstetrícia. Diretrizes clínicas na saúde suplementar - câncer de mama: terapia endócrina e terapia alvo. https://diretrizes.amb.org.br/ (acessado em 16/Fev/2020). 
25. Associação Médica Brasileira; Agência Nacional de Saúde Suplementar; Sociedade Brasileira de Cancerologia. Diretrizes clínicas na saúde suplementar - câncer de cólon: tratamento quimioterápico. https://diretrizes.amb.org.br/ (acessado em 16/Fev/2020).

26. Associação Médica Brasileira; Sociedade Brasileira de Urologia. Adenocarcinoma Metastático de Próstata resistente à castração. https://diretrizes.amb.org.br/ (acessado em 16/Fev/2020).

27. Unimed do Brasil - Confederação Nacional das Cooperativas Médicas. Tratativa Oncológica 2016. https://www.unimed.coop.br/ documents/1262603/3098105/\%28ANEXO $\% 29+1+-+$ Tratativa+Oncologia_WEB+2016. pdf/36f772cd-5b69-4252-86a1-76c0f832c4e5 (acessado em 16/Fev/2020).

28. Sociedade Brasileira de Oncologia Clínica. Carcinoma de mama. https://www.sboc. org.br/images/diretrizes/diretrizes_pdfs/ Carcinoma_de_Mama.pdf (acessado em 16/ Fev/2020).

29. Sociedade Brasileira de Oncologia Clínica. Câncer de próstata. https://www.sboc.org.br/ images/diretrizes/Câncer_de_próstata_2018. pdf (acessado em 16/Fev/2020).

30. Sociedade Brasileira de Oncologia Clínica. Adenocarcinoma colorretal. https://www. sboc.org.br/images/diretrizes/diretrizes_ pdfs/Adenocarcinoma_de_Colorretal.pdf (acessado em 16/Fev/2020).

31. Departamento de Gestão e Incorporação de Tecnologias em Saúde, Secretaria de Ciência, Tecnologia e Insumos Estratégicos, Ministério da Saúde. Diretrizes metodológicas - elaboração de diretrizes clínicas. https://www.inca. gov.br/bvscontrolecancer/publicacoes/Rela torio_Diretrizes_CP_04_2016.pdf (acessado em $16 /$ Fev/2020).

32. Departamento de Gestão e Incorporação de Tecnologias em Saúde, Secretaria de Ciência, Tecnologia e Insumos Estratégicos, Ministério da Saúde. Guia de elaboração: escopo para Protocolos Clínicos e Diretrizes Terapêuticas. 2a Ed. Brasília: Ministério da Saúde; 2019.

33. Brouwers MC, Kerkvliet K, Spithoff K; AGREE Nextt Steps Consortium. The AGREE reporting checklist: a tool to improve reporting of clinical practice guidelines. BMJ 2016; 352:i1152.

34. Ministerio de Salud y Protección Social. Guías de práctica clínica GPC. http://gpc.minsalud. gov.co/gpc (acessado em 16/Fev/2020).

35. Scottish Intercollegiate Guidelines Network. Our guidelines. https://www.sign.ac.uk/ourguidelines (acessado em 16/Fev/2020).

36. Reis EC, Passos SRL, Santos MAB. Quality assessment of clinical guidelines for the treatment of obesity in adults: application of the AGREE II instrument. Cad Saúde Pública 2018; 34:e00050517.

37. Burda BU, Chambers AR, Johnson JC. Appraisal of guidelines developed by the World Health Organization. Public Health 2014;128:444-74.
38. Brasil. Decreto no 7.508 , de 28 de junho de 2011. Regulamenta a Lei no 8.080, de 19 de setembro de 1990, para dispor sobre a organização do Sistema Único de Saúde - SUS, o planejamento da saúde, a assistência à saúde e a articulação interfederativa, e dá outras providências. Diário Oficial da União 2011; 29 jun.

39. Comissão Nacional de Incorporação de Tecnologias no Sistema Único de Saúde. Protocolos e Diretrizes do Ministério da Saúde. http:// conitec.gov.br/pcdt-em-elaboracao (acessado em 16/Fev/2020).

40. Ministério da Saúde. Portaria no 25, de 22 de abril de 2019. Torna pública a decisão de não incorporar o acetato de ciproterona em pacientes com adenocarcinoma de próstata resistente à castração, no âmbito do Sistema Único de Saúde - SUS. Diário Oficial da União 2019; 23 abr.

41. Ministério da Saúde. Portaria no 38, de 24 de julho de 2019. Torna pública a decisão de incorporar a abiraterona para o câncer de próstata metastático resistente à castração de pacientes com uso prévio de quimioterapia, conforme a Assistência Oncológica no SUS, no âmbito do Sistema Único de Saúde - SUS. Diário Oficial da União 2019; 25 jul.

42. Abdelsattar ZM, Reames BN, Regenbogen SE, Hendren S, Wong SL. Critical evaluation of the scientific content in clinical practice guidelines. Cancer 2015; 121:783-9.

43. Caetano R, Silva RM, Pedro EM, Oliveira IAG, Biz AN, Santana P. Incorporação de novos medicamentos pela Comissão Nacional de Incorporação de Tecnologias do SUS, 2012 a junho de 2016. Ciênc Saúde Colet 2017; 22:2513-25.

44. Jacobs C, Graham ID, Makarski J, Chassé M, Fergusson D, Hutton B, Clemons M. Clinical practice guidelines and consensus statements in oncology - an assessment of their methodological quality. PLoS One 2014; 9:e110469.

45. Wayant C, Cooper C, Turner D, Vassar M. Evaluation of the NCCN guidelines using the RIGHT Statement and AGREE-II instrument: a cross-sectional review. BMJ Evid Based Med 2019; 24:219-26.

46. Guyatt G, Oxman AD, Akl EA, Kunz R, Vist G, Brozek J, et al. GRADE guidelines: 1. Introduction - GRADE evidence profiles and summary of findings tables. J Clin Epidemiol 2011; 64:383-94.

47. National Institute of Clinical Excellence. Developing NICE guidelines: the manual - process and methods. https://www.nice.org.uk/ process/pmg20/resources/developing-niceguidelines-the-manual-pdf-72286708700869 (acessado em 16/Fev/2020).

48. Tyagi NK, Dhesy-Thind S. Clinical practice guidelines in breast cancer. Curr Oncol 2018; 25 Supp 1:S151-60. 
49. Gagliardi AR, Brouwers MC. Do guidelines offer implementation advice to target users? A systematic review of guideline applicability. BMJ Open 2015; 5:e007047.

50. Augustsson H, Churruca K, Braithwaite J. Re-energising the way we manage change in healthcare: the case for soft systems methodology and its application to evidence-based practice. BMC Health Serv Res. BMC Health Serv Res 2019; 19:666.

51. Rocque GB, Williams CP, Kenzik KM, Jackson BE. Concordance with NCCN treatment guidelines: relations with health care utilization, cost, and mortality in breast cancer patients with secondary metastasis. Cancer 2018; 124:4231-40.

52. Senkus E, Lacko A. Over-treatment in metastatic breast cancer. Breast 2017; 31:309-17.

53. Kaliks RA, Matos TF, Silva VDA, Holtz L, Barros DC. Diferenças no tratamento sistêmico do câncer no Brasil: meu SUS é diferente do teu SUS. Braz J Oncol 2017; 13:1-12.
54. Agência Nacional de Vigilância Sanitária. RDC no 200, de 26 de dezembro de 2017. Dispõe sobre os critérios para a concessão e renovação do registro de medicamentos com princípios ativos sintéticos e semissintéticos, classificados como novos, genéricos e similares, e dá outras providências. Diário Oficial da União 2017; 29 jan.

55. Chen EY, Raghunathan V, Prasad V. An overview of cancer drugs approved by the US Food and Drug Administration based on the surrogate end point of response rate. JAMA Intern Med 2019; 179:915-21.

56. Gyawali B, Hey SP, Kesselheim AS. Assessment of the clinical benefit of cancer drugs receiving accelerated approval. JAMA Intern Med 2019; 179:906-13.

57. Ministério da Saúde. Portaria Conjunta no 5, de 18 de abril de 2019. Aprova as Diretrizes Diagnósticas e Terapêuticas do Carcinoma de Mama. Diário Oficial da União 2019; 29 abr. 


\section{Abstract}

The expansion in the variety of clinical guidelines in oncology is perceptible worldwide, highlighting the need to guarantee the quality of these documents. The study thus aimed to assess the quality of Brazilian national guidelines for treatments of breast, prostate, and colon and rectal cancers. We selected 12 Brazilian guidelines published by four different drafting groups (Ministry of Health, Supplementary Health System, and medical societies and associations), and the AGREE II instrument was applied. In all these guidelines, we identified important weaknesses in more than one Domain, especially low values for "applicability" and "editorial independence". The patterns observed per Domains are more related to the drafting group than the respective clinical conditions. Lower scores in "drafting rigor" and "editorial independence" were obtained by nongovernmental drafting groups, including absence of information or lack of its transparency. Although the "clarity of presentation" in the Ministry of Health guidelines was relatively lower, all the guidelines presented major limitations in "applicability". Consequently, in the overall assessment, none of the guidelines was recommended without modifications, and four were not recommended at all. Finally, it is necessary to upgrade the guidelines according to the underlying evidence ("methodological rigor") and to present the recommended practices in a comprehensible and applicable way ("applicability"), and to mitigate conflicting interests in order to offer cancer patients the best available care in Brazil.

Practice Guideline; Medical Oncology; Biomedical Technology Assessment

\section{Resumen}

La ampliación de la variedad de directrices clínicas en oncología es perceptible en todo el mundo, lo que resalta la necesidad de garantizar la calidad de estos documentos. Por este motivo, el objetivo de este estudio fue evaluar la calidad de directrices nacionales de tratamientos de los cánceres de mama, próstata, así como de colon y recto. Se seleccionaron doce directrices brasileñas, publicadas por cuatro grupos elaboradores distintos (Ministerio de Salud, sistema suplementario de salud, así como sociedades y asociaciones médicas), y se aplicó el instrumento AGREE II. En todas las directrices evaluadas se identificaron debilidades importantes en más de un Dominio, destacando los bajos valores para la "aplicabilidad" e "independencia editorial". Los patrones observados por Dominios se presentan más relacionados con el grupo elaborador, que con las condiciones clínicas tratadas. Menores puntuaciones en el "rigor del desarrollo" $e$ "independencia editorial" se obtuvieron por parte de grupos elaboradores no gubernamentales, inclusive con ausencia of falta de transparencia en la información. A pesar de que la "claridad de la presentación" de las directrices del Ministerio de la Salud haya sido relativamente inferior en la "aplicabilidad", todas presentaron limitaciones expresivas. Consecuentemente, en la evaluación global, ninguna de las directrices fue recomendada sin modificaciones, siendo cuatro de ellas no recomendadas. Por fin, se hace necesario cualificar las recomendaciones, en lo que se refiere a las evidencias que las fundamentan ("rigor metodológico"), así como disponer de forma comprensible y factible las conductas que deben ser adoptadas ("aplicabilidad"), al igual que mitigar intereses conflictivos, para que sea ofertado el mejor cuidado a los pacientes oncológicos en el país.

Guías de Práctica Clínica; Oncología Médica; Evaluación de la Tecnología Biomédica

Recebido em 10/Fev/2020

Versão final reapresentada em 11/Mai/2020

Aprovado em 20/Ago/2020 